\title{
¿Son útiles las novelas policíacas como instrumento docente en farmacología?
}

\author{
Josep Eladi Baños, Magí Farré
}

Las novelas policíacas se han asociado con la medicina desde casi su inicio en la historia de la literatura. A pesar de que algunos autores consideran a Edgard Allan Poe como el creador de su versión más moderna [1], sería más justo reconocer la contribución del oftalmólogo escocés Arthur Conan Doyle en el inicio del género tal como lo conocemos en la actualidad [2]. Sherlock Holmes, el protagonista de sus novelas, y su compañero, el doctor Watson, conforman el modelo sobre el que se construirían numerosas obras del pasado siglo. De hecho, se ha sugerido incluso que el proceso deductivo propio de los detectives de tales novelas tenía mucho que ver con la lógica aplicada al diagnóstico por los médicos [3]. Quizá por ello, la nómina de los médicos que se han dedicado a este género no es desdeñable. Recuérdese, entre los clásicos, a Robert Austin Freeman (1892-1943) y Doris Bell Ball (1897-1987), más conocida por su pseudónimo, Josephine Bell. Entre los más recientes destacan Leonard Goldberg, Robin Cook, Michael Crichton (1942-2008) y Tess Garritsen [2]. En España también hay ejemplos de profesionales sanitarios que han cultivado la novela policíaca, como los médicos Lluís Daufí [4] (1929-2013), Alejandro Arís [5], Amàlia Lafuente y Carles Martín Fumadó [5], así como el farmacéutico Carlos Lens.

Numerosas publicaciones sugieren que la literatura puede contribuir a la formación de los médicos al proporcionarles una visión más holística del proceso de enfermedad [6,7]. Enlazando ambos temas, nos preguntamos si las novelas policíacas podrían constituir un instrumento de interés para ayudar a los estudiantes a comprender mejor la farmacología. De hecho, el empleo de tales obras ya se ha sugerido en otras disciplinas, como la química [8].

Para explorar la viabilidad de esta hipótesis, hemos revisado varias obras que analizan la literatura policíaca de ficción $[1,9,10]$, así como las obras de varios autores clásicos (Arthur Conan Doyle, Agatha Christie) y modernos (Donna Leon, Henning Mankell, Andrea Camilleri, Robin Cook, Michael Crichton) de este género literario. Este proceso nos ha permitido alcanzar algunas conclusiones importantes. En primer lugar, en la mayoría de las novelas, los fármacos se utilizan con intención homicida. En segundo, muchos de los fármacos empleados deberían calificarse más como venenos que como medicamentos, como es el caso de la estricnina o el arsénico; aunque ambos se utilizaron en el pasado en la composición de preparados terapéuticos [10, 11], en la actualidad su interés es meramente toxicológico, aunque algunos de ellos, como el cianuro, tienen interés para explicar procesos fisiológicos. En tercer lugar, existen fármacos que se emplean en terapéutica contemporánea y que están presentes en las novelas policíacas, entre ellos los opioides, los aminoglicósidos, los digitálicos, los anticolinesterásicos, los antimuscarínicos, los barbitúricos, la ketamina e incluso la toxina botulínica.

Los hallazgos citados confirman el interés preliminar de los relatos policíacos para estimular el interés de los estudiantes de medicina en saber cómo los fármacos ejercen sus efectos y para facilitar el conocimiento de las interacciones complejas entre sustancias químicas y seres vivos. Varios ejemplos pueden contribuir a ilustrar esta opinión. Por ejemplo, en Sad cypress (1940), de Agatha Christie, se muestra cómo la intoxicación con morfina puede evitarse con apomorfina. En The adventure of Sussex vampire (1924), de Arthur Conan Doyle, se describe una intoxicación accidental con curare. En Death at la Fenice (1992), de Donna Leon, se describe cómo la administración repetida de netilmicina produce sordera a un director de orquesta, lo que le conduce a un suicidio simulado. En La llave de la felicidad
Departamento de Ciencias Experimentales y de la Salud; Universitat Pompeu Fabra (J.E. Baños). Departamento de Farmacología, Terapéutica y Toxicología; Universitat Autònoma de Barcelona (M. Farré). Instituto de Investigación Médica, IMIM; Hospital del Mar (M. Farré). Barcelona, España.

Correspondencia: Dr. Josep Eladi Baños. Departamento de Ciencias Experimentales y de la Salud. Universitat Pompeu Fabra. Doctor Aiguader, 88. E-08003 Barcelona.

E-mail: josepeladi.banos@upf.edu (c) 2015 FEM 
(2005), de Lluís Daufí, la trama discurre alrededor de unos derivados de heroína y morfina que desatan una serie de asesinatos. También serían de interés obras más recientes, como Terapia de riesgo (2013), de Amàlia Lafuente, sobre medicamentos biotecnológicos y la ética de su obtención, y El peor de los venenos, de Carlos Lens, sobre el tenebroso mundo de la falsificación de medicamentos.

En conclusión, consideramos que las historias policíacas de ficción pueden suponer un complemento interesante en la docencia de la farmacología y, probablemente, de otras disciplinas. Una cuestión importante es cómo implementarlas dentro de los planes de estudio actuales. En nuestra opinión existen varias posibilidades: convertir la lectura de las novelas en actividades obligatorias de los planes docentes, utilizar fragmentos ilustrativos o aprovechar las obras para escribir textos que puedan emplearse en actividades de grupo reducido (seminarios, aprendizaje basado en problemas). Cada profesor debería escoger la circunstancia que mejor se adapta a su entorno docente. Por supuesto, la eficacia de esta propuesta debería demostrarse con estudios empíricos, pero la experiencia positiva con textos literarios en otros ámbitos [12-14] justifica, al menos, una prueba.
Bibliografía

1. Velasco-Martín A. Los venenos en la literatura policíaca. Valladolid: Universidad de Valladolid; 2011.

2. Baños JE, Torrens M. Novel.la negra i metges: exemples d'una associació gens aleatòria. Ann Med 2012; 95: 122-7.

3. Rapezzi C, Ferrari R, Branzi A. White coats and fingerprints: diagnostic reasoning in medicine and investigative methods of fictional detectives. BMJ 2005; 331: 1491-4.

4. Baños JE, Torrens M. Un altre escriptor de novel-la políciaca: Lluís Daufí i Moreso. Ann Med 2013; 96: 191.

5. Baños JE, Torrens M. Novel-la negra i metges catalans: dos exemples. Ann Med 2013: 96: 96.

6. Hunter KM, Charon R, Coulehan JL. The study of literature in medical education. Acad Med 1995; 70: 787-94.

7. Baños JE. El valor de la literatura en la formación de los estudiantes de medicina. Educ Med 2003; 6: 93-9.

8. Labianca DA, Reeves WJ. Chemistry and detective fiction. An interdisciplinary program for the nonscience major. J Chem Educ 1981; 58: 683-6.

9. Gerald MC. The poisonous pen of Agatha Christie. Austin: University of Texas Press; 1993.

10. Emsley J. The elements of murder. A history of poison. Oxford: Oxford University Press; 2005.

11. Whorton JC. The arsenic century. How Victorian Britain was poisoned at home, work, and play. Oxford: Oxford University Press; 2010.

12. Behrendt S, ed. Approaches to teaching Mary Shelley's Frankenstein. New York: The Modern Language Association of America; 1990.

13. Brungardt G. Teaching The death of Ivan Ilych: a guide to introducing Tolstoy's classic. J Palliative Med 2009; 12: 679-82.

14. Kerr L. Always the same story: familiar narrative structures in Oliver Sacks and Nancy Mairs. Fam Med 2010; 42: 97-9. 